\title{
Improving mental health services beyond COVID-19
}

\author{
E. Power ${ }^{1,2}$ and J. Lyne $\mathrm{P}^{1,3, *}$ \\ ${ }^{1}$ Department of Psychiatry, Royal College of Surgeons in Ireland, Dublin, Ireland \\ ${ }^{2}$ Department of Liaison Psychiatry, Beaumont Hospital, Dublin, Ireland \\ ${ }^{3}$ Newcastle Hospital, Greystones, Co. Wicklow, Ireland
}

In this September 2021 issue of the Irish Journal of Psychological Medicine, several topical, clinical, and service-related issues are comprehensively explored. The issue contains articles related to several themes, including COVID-19, which is likely to remain a theme in mental health research in the short to medium term.

Bourke et al. (pp 169-176) examine resource utilization costs in borderline personality disorder in Ireland. Using prevalence-based cost analysis, they estimate costs of $€ 10844$ annually. This was less spend than in equivocal comparator studies in Western Europe. Almost two-thirds of costs relate to inpatient care and medication - of which neither can be considered first line evidence-based treatment recommendations for borderline personality disorder. Such lack of spend and disproportionality in spend is a serious concern regarding the care delivered for individuals living with borderline personality disorder, an eminently treatable condition. In addition, this study did not estimate indirect or other costs, such as economic losses, suggesting that the absolute costs of borderline personality disorder to society could be higher again.

In their paper, $\mathrm{O}^{\prime}$ Neill et al. provide a re-examination of the Penrose hypothesis (pp 182-185). Much data have reported a correlation between deinstitutionalization (by measuring available psychiatric hospital beds) and increasing prison population places. In this paper, it is demonstrated that the Penrose hypothesis is supported when examining this issue using psychiatric hospital admissions and prison committals. As is eloquently delineated in the manuscript, there are several reasons to suspect that limitations in the community treatment of mental disorders more broadly are causally related to increased prison population numbers. Making more robust causal inferences from such ecological datasets, however, is elusive, and the authors outline methods for investigating this further.

The human rights imperative for diverting individuals experiencing mental disorder away from the

\footnotetext{
*Address for correspondence: Dr J. Lyne, Newcastle Hospital, Greystones, Co. Wicklow, Ireland.

(Email: johnlyne@rcsi.ie)
}

criminal justice system remains an important policy consideration. In their letter in this issue, Gulati et al. (pp. 232-233) also outline the crucial importance of maintaining healthcare standards and human rights for prisoners during the COVID-19 pandemic.

Patel \& Yacoub (pp 177-181) examine rates of diagnosis of attention deficit hyperactivity disorder (ADHD) in individuals with intellectual disability attending a community intellectual disability (ID) service. Overall point prevalence was in keeping with estimates in previous general population studies, although the adult rate of ADHD diagnosis was at the lower end of the literature range. In contrast, the child rate of ADHD diagnosis was at the higher end of the range, with a notable figure of $50 \%$ of children with mild ID also having a diagnosis of ADHD.

In their editorial, Roche \& Russell (pp 163-168) discuss precision medicine, a field which considers differences in genes, environments, and lifestyles in an effort to tailor treatments to individual patients. Personalized and precision psychiatry is progressing in both clinical and research practice with much potential for improving clinical outcomes. In spite of the challenges of precision medicine, such advances would be very welcome particularly in the context of developing novel drug and therapeutic approaches.

There are four book reviews in this issue highlighting some very interesting reading for psychiatrists and mental health service users alike. Colquhoun (pp 238-239) provides a glowing review of a comprehensive book about therapies in 'Treatments for Psychological Problems and Syndromes'. Kelly (pp 234-235) hooks us with an intriguing opening paragraph of his review of 'The Selected Writings of Maurice O'Connor Drury on Wittgenstein, Philosophy, Religion and Psychiatry'. Wittgenstein's fascinating Irish connection with psychiatrist $\mathrm{O}^{\prime}$ Connor Drury and his status as one of the finest philosophers of the 20th century do, indeed, denote this as an important reading. Gulati \& Mulryan enthusiastically review 'The Psychobiotic Revolution: Mood, Food and the New Science of the Gut-Brain Connection'. They highlight the book as an accessible read that strikes both a persuasive and a balanced tone. 
Finally, Bredin (pp 236-237) reviews 'In My Room', by Professor Jim Lucey, which delivers insights for readers about the journey toward recovery and how this journey differs across individual experiences.

The effects of the COVID-19 pandemic have invigorated discussion on the mental health of the population and drawn scrutiny to the state of mental health services from many perspectives. $\mathrm{O}^{\prime}$ Reilly et al. (pp 192-207) present a comprehensive rapid review of the impact of pandemics on youth mental health. Their synthesis of studies considers how we can support young people during future pandemic events. Mahyijari et al. explore the psychological impact among healthcare workers who had close contact with COVID19 patients in the Sultanate of Oman. They found concerning levels of anxiety and stress as well as suboptimal well-being reminding us of the severe stresses experienced by frontline workers during the pandemic. Meagher et al. (pp 208-213) give us their perspective on managing COVID-19-associated delirium, providing a valuable summary of potential drug-drug interactions among other considerations. Frawley et al. (pp 220-226) provide a unique perspective of the pandemic from a mental health nursing viewpoint. Their paper takes a person-centered approach, and the supplementary material for the paper includes very interesting vignettes from both nursing and patient perspectives.

Finally, O' Donoghue \& Colleagues (pp 214-219) write a defining perspective piece on COVID-19 and early intervention in psychosis (EIP). EIP services in Ireland are at a nascent stage of delivery on a national scale, and the authors give credible arguments on why EIP rollout must continue. Echoing the authors call to action, the editorial team also believe we can and must deliver advanced 21st century standards of mental healthcare.

\section{Conflict of interest}

The authors have no conflicts of interest to declare. 